\title{
Path Planning of Robotic Fish Based on Fuzzy Control
}

\author{
Xin Li, Yansong Deng * \\ College of Electrical and Information Engineering, Southwest University for Nationalities \\ Chengdu, Sichuan, 610041, P. R. China \\ *Corresponding author e-mail:dengyansong@gmail.com
}

\begin{abstract}
Due to the complexity and the uncertainty of water environment, the majority of communal gorithms of local path planning are almost approximate and bring some noise interference, so it is difficult to control accurately. Referring to the idea of fuzzy control to solve problems of the robotic fish collide, the thesis makes use of the robustness of fuzzy control and the timing adjustment of deflection angle of the robotic fish with the biology combination of perception and action, and reaches the target point effectively. The algorithm possesses a better avoidance capability in multi obstacle and complex cases. Further more, it is simple and easy to carry out.
\end{abstract}

Keywords-fuzzy control; robotic fish; path planning; URWPGSim2D.

\section{INTRODUCTION}

Recently, with the rapid development of biological engineering and technical engineering, people have shown an increasing interest in the biological movement mechanism. Therefore, the study on the robotic fish emerges as the times require. URWPGSim2D of the robotic fish truly simulate the fluctuation of water situation. It provides the theoretical foundation and experiment platform for our further research through the robotic fish simulating the complex movement mechanism and completing various projects in the URWPGSim2D.

The research of the robotic fish involves so many aspects, and the path planning is one of the most significant research fields. The common path planning algorithms are: Wang $\mathrm{Na}$ [1] proposed a path planning algorithm based on mesh model, which searched for a safe path through the grid analysis modeling; Jin Jiucai [2] used a hierarchical genetic algorithm for path planning of underwater robot through the establishment of environmental model, but the disadvantage of that was the method cannot well solve amounts of calculations problems; Ernesto P L [3] established the obstacle avoidance strategy based on artificial potential field method, which effectively navigated mobile robots, but this method easily fell into local minimum point.

The paper proposes a fuzzy control algorithm for fish robot obstacle avoidance. The algorithm, according to the specific circumstances of the controlled object, makes the path planning for the underwater robotic fish. It just needs to take system control of the object according to the knowledge and experience rather than to establish the precisely mathematical model. Simulation experiments prove that the robotic fish based on this fuzzy control algorithm can reach the target point safely and reliably without collision.

\section{OPERATION PRINCIPLES}

With the hypothesis the robotic fish in water of uniform motion, it can timely measure the nearest distance ' $d$ ' between the robotic fish and the obstacle, and the angle ' $\theta$ ', among the obstacle, the robotic fish, andtarget, through the short baseline system. With the fuzzy control algorithm of reasoning, it can calculate the steering angle that the underwater robotic fish requires in obstacle avoidance condition, so the robotic fish avoids obstacles safely, and reach the target location accurately [4]. Specific parameters' description is shown in figure 1.

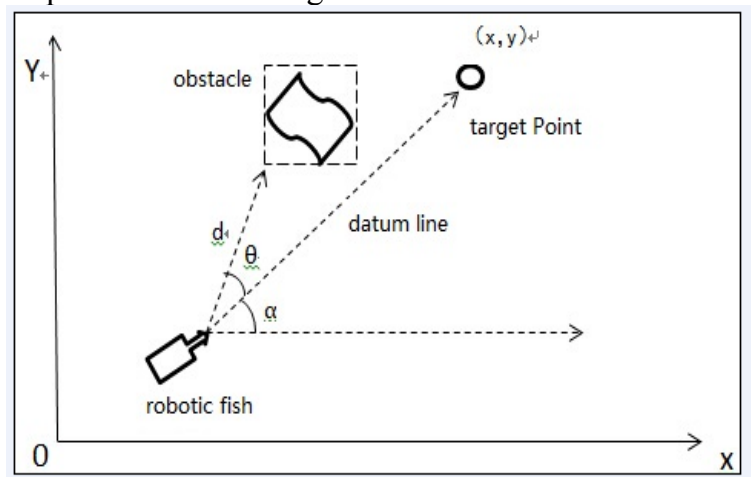

Figure 1. The principle diagram of the navigation

\section{A. The distance $d$ calculated}

If the size of the obstacle is tiny, the size or shape can be negligible, according to two direct calculation of distance:

$$
d=\sqrt{\left(\mathrm{x}-x_{1}\right)^{2}+\left(\mathrm{y}-y_{1}\right)^{2}}
$$

When the obstacle is the center of a circle, circle center coordinate set is $[\mathrm{x} 0, \mathrm{y} 0]$, radius is $\mathrm{R}$, and the distance to the obstacle robotic fish:

$$
d=\sqrt{\left(\mathrm{x}-x_{0}\right)^{2}+\left(\mathrm{y}-y_{0}\right)^{2}}-\mathrm{r}
$$

If the barrier is large with less rules, it can use the method of approximated transformation, regarding the disorder as a rectangle to calculate distance.Specific ideas are as follows: 


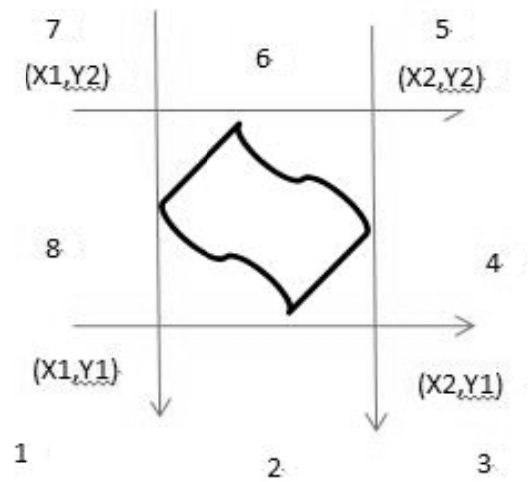

Figure 2. Schematic diagram of minimum distance between obstacles and the robotic fish

When the robotic fish is located in 1 area, namely $\mathrm{x}<\mathrm{x} 1, \mathrm{y}<\mathrm{y} 1$, the distance coordinates of the shortest distance is $(\mathrm{x} 1, \mathrm{y} 1)$; When the robotic fish is located in 2 area, namelyx $1<\mathrm{x}<\mathrm{x} 2, \mathrm{y}<\mathrm{y} 1$, the distance coordinates of the shortest distance is $(\mathrm{x}, \mathrm{y} 1)$; When the robotic fish is located in 3 area, namely $x>x_{2}, y<y_{1}$, the distance coordinates of the shortest distance is $(x 2, y 1)$; When the robotic fish is located in 4 area, namely $\mathrm{x}>\mathrm{x}_{2}, \mathrm{y}_{1}<\mathrm{y}<\mathrm{y}_{2}$, the distance coordinates of the shortest distance is $(\mathrm{x} 2, \mathrm{y})$; When the robotic fish is located in 5 area, namely $\mathrm{x}>\mathrm{x}_{2}, \mathrm{y}>\mathrm{y}_{2}$, the distance coordinates of the shortest distance is $(\mathrm{x} 2, \mathrm{y} 2)$; When the robotic fish is located in 6 area, namely $\mathrm{x}_{1}<\mathrm{x}<\mathrm{x}_{2}, \mathrm{y}>\mathrm{y}_{2}$, the distance coordinates of the shortest distance is $(\mathrm{x}, \mathrm{y} 2)$; When the robotic fish is located in 7 area, namely $\mathrm{x}<\mathrm{x}_{1}, \mathrm{y}>\mathrm{y}_{2}$, the distance coordinates of the shortest distance is $(\mathrm{x} 1, \mathrm{y} 2)$; When the robotic fish is located in 8 area, namely $\mathrm{x}<\mathrm{x}_{1}, \mathrm{y}_{1}<\mathrm{y}<\mathrm{y}_{2}$.the distance coordinates of the shortest distance is $(\mathrm{x} 1, \mathrm{y})$.

The distance between the robotic fish and rectangular obstacle transfers into the shortest distance between the fish and the rectangular box machine:

$$
d=\sqrt{\left(\mathrm{x}-x_{d}\right)^{2}+\left(\mathrm{y}-y_{d}\right)^{2}}
$$

\section{B. Solving the angle $\theta$}

Use the link between the robotic fish and target as the baseline; calculate the angle $\theta$ [5] by coordinates of the obstacle, the obstacle, and the robotic fish; the angle $\theta$ on the left side of the baseline is positive; it is negative on the right.

$$
\begin{aligned}
& \theta=\arccos \frac{\mathrm{a}^{2}+\mathrm{b}^{2}+\mathrm{c}^{2}}{2 \mathrm{ab}} \\
& \theta=-\arccos \frac{\mathrm{a}^{2}+\mathrm{b}^{2}+\mathrm{c}^{2}}{2 \mathrm{ab}}
\end{aligned}
$$

In the formula: $a$ is the nearest distance between the machine fish and obstacles; $b$ is the distance between target point and the machine fish; $c$ is the distance between the obstacle nearest point and the target point.

\section{THE DESIGN OF FUZZY CONTROLLER}

Fuzzy control makes use of the control law described by fuzzy mathematics language (control rules) to manipulate the system work. Control device according to fuzzy control law is known as the fuzzy controller. In practical engineering, many systems and processes are very complex, and it is difficult to establish precisely mathematical model and design the general controller. Usually, the only way is that skilled operatormanually takes control by their experience. Thus, the control rule is often based on Fuzzy form reflecting on control personnel experience, and it is difficult to use the traditional mathematical language to describe.

\section{A. Select the input and output variables of the fuzzy controller}

The fuzzy logic algorithm combines the inherent robustness of fuzzy control and the 'perception -- action' based on biology, which puts forward the new idea of the robotic fish's underwater actions [6]. On the basis of fuzzy reasoning's front piece and back piece, namely the input and output of the machine inference, select the distance $d$ between fish and obstacle, and the $\operatorname{target} \theta$ between the fish and relative to the machine connection as input variables of the controller, select the robotic fish with the rotation angle $\Phi$ as the output of fuzzy controller.

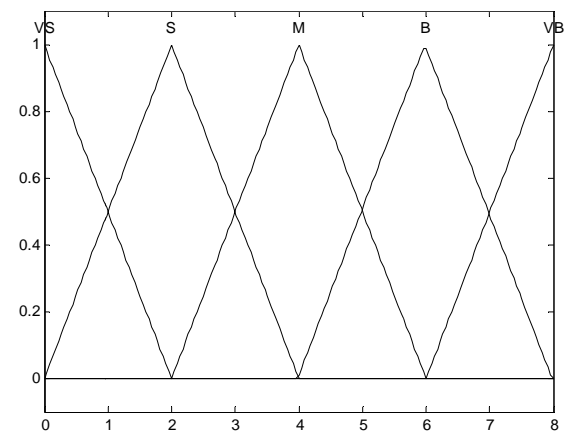

Figure 3. The fuzzy segmentation graph ofdistanced

TABLE I. THE MEMBERSHIP FUNCTION OFd DISTANCE

\begin{tabular}{cccccccccc}
\hline$\theta(\phi)$ & -4 & -3 & -2 & -1 & 0 & 1 & 2 & 3 & 4 \\
\hline $\mathrm{NL}$ & 1.0 & 0.5 & 0.0 & 0.0 & 0.0 & 0.0 & 0.0 & 0.0 & 0.0 \\
$\mathrm{NS}$ & 0.0 & 0.5 & 1.0 & 0.5 & 0.0 & 0.0 & 0.0 & 0.0 & 0.0 \\
$\mathrm{Z}$ & 0.0 & 0.0 & 0.0 & 0.5 & 1.0 & 0.5 & 0.0 & 0.0 & 0.0 \\
$\mathrm{PS}$ & 0.0 & 0.0 & 0.0 & 0.0 & 0.0 & 0.5 & 1.0 & 0.5 & 0.0 \\
$\mathrm{PL}$ & 0.0 & 0.0 & 0.0 & 0.0 & 0.0 & 0.0 & 0.0 & 0.5 & 1.0 \\
\hline
\end{tabular}

\section{B. The fuzzy membership function}

Considering the ability of the robotic fish to change its direction, the value of distance $d$ betweenthe simulated robotic fish and obstacle ranges from $[0,80]$ to $[0,8]$. The quantization interval, a fuzzy domain sub file is $\{0,1,2,3,4,5,6,7,8\}$, and inputlanguage value of $d$ is very small (VS), small (S), (M), high (B), large (VB).

The angle $\theta$ range of robotic fish and the obstacle of $\left[-90^{\circ},+90^{\circ}\right]$, quantization to $[-3,3]$ interval, a fuzzy domain 
sub file for $[-4,-3,-2,-1,0,1,2,3,4]$, language value of $\theta$ was Negative Large (NL), Negative Small (NS), zero (O), Positive Small (PS), Positive Large (RL).

The range of robotic fish with deflection angle of $\left[-45^{\circ}\right.$, $\left.+45^{\circ}\right]$, quantization to $[-3,3]$ interval, a fuzzy domain sub file for $[-3,-2,-1,0,1,2,3]$, language value of angleawas Negative Large (NL), Negative Small (NS), zero (O), Positive Small (PS), Positive Large (PL).

Through the fuzzy input and output domain sub file to set the appropriate value of fuzzy language, it uses the fuzzy triangular membership function of fuzzy to set to reaction[7]. Fuzzy segmentation graphics and membership function of distance $d$ show in Figure 3 and table 1,fuzzy segmentation graphics and membership function of angle $\theta(\Phi)$ show in Figure 4 and table 2 shows.

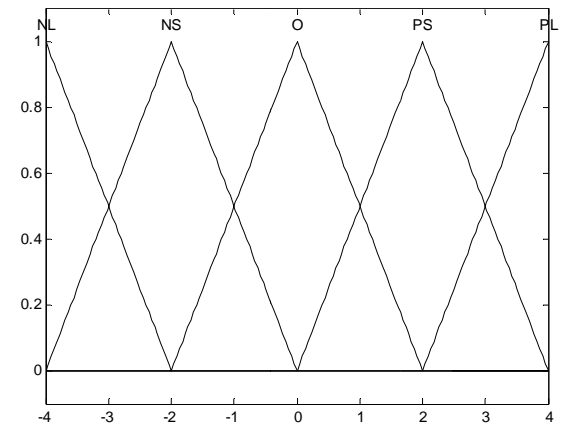

Figure 4. The fuzzy segmentation graph of angle $\theta(\Phi)$

TABLE II. THE MEMBERSHIP FUNCTION OF $\theta(\Phi)$

\begin{tabular}{cccccccccc}
\hline$d$ & 0 & 1 & 2 & 3 & 4 & 5 & 6 & 7 & 8 \\
\hline VS & 1.0 & 0.5 & 0.0 & 0.0 & 0.0 & 0.0 & 0.0 & 0.0 & 0.0 \\
S & 0.0 & 0.5 & 1.0 & 0.5 & 0.0 & 0.0 & 0.0 & 0.0 & 0.0 \\
M & 0.0 & 0.0 & 0.0 & 0.5 & 1.0 & 0.5 & 0.0 & 0.0 & 0.0 \\
B & 0.0 & 0.0 & 0.0 & 0.0 & 0.0 & 0.5 & 1.0 & 0.5 & 0.0 \\
VB & 0.0 & 0.0 & 0.0 & 0.0 & 0.0 & 0.0 & 0.0 & 0.5 & 1.0
\end{tabular}

\section{The establishment of fuzzy rules}

The basic principles for the robotic fish to avoid obstacles is: when the obstacle distance is greater than a certain value, the robotic fish moves forward to the target; when obstacles is on the left side of the reference line, it turns right to avoid obstacles; when obstacles is on the right side of the reference line, it turns left; when the obstacle is too close to it, the robotic fish turns right by default. As the number of the fuzzy set elements of the fuzzy controller input variable $\theta$ is 5 ; the input variables of $d$ fuzzy sets is 5 ; combined with the experience, the number of establishment of fuzzy control rules is 25 ; it has the following form of fuzzy rules:

If $\mathrm{d}$ is $\mathrm{A}_{1}$ and $\theta$ is $\mathrm{B}_{1}$, then $\Phi$ is $\mathrm{C}_{11}$

If $\mathrm{d}$ is $\mathrm{A}_{1}$ and $\theta$ is $\mathrm{B}_{2}$, then $\Phi$ is $\mathrm{C}_{12}$

If $d$ is $A_{i}$ and $\theta$ is $B_{j}$, thenФis $C_{i j}$

Table 3 shows the fuzzy control rule.
TABLE III. THE RULE TABLE OF FUZZY CONTROL

\begin{tabular}{cccccc}
\hline & NL & NS & Z & PS & PL \\
\hline VS & NL & NL & NL & PL & PL \\
S & NL & NL & NL & PL & PL \\
M & NS & NS & Z & PS & PS \\
B & NS & Z & Z & Z & PS \\
VB & NS & Z & Z & Z & PS \\
\hline
\end{tabular}

D. The path planning process is as follows:

1) Initialize the data, including the starting point coordinates of the robotic fish, moving step size, type, the obstacle of initial coordinate, and coordinates of target;

2) Determine whether the coordinate of the robotic fish itself is the target coordinate or not; "NO" means to continue; "YES" means to do the eighth step;

3) Calculate the shortest distance ' $d$ ' of the robotic fish and obstacles;

4) Determine whether $d$ is greater than the minimum influence distance 'dmin'; "NO", continue to run; "YES" moves to the seventh step;

5) Calculate the angle $\theta$ among the obstacle points, the robotic fish and the target point of attachment; then judge it;

6) Inputdand $\theta$ into the fuzzy controller[8], and use the center of area method[9] to get fuzzy, in order to get the needed deflection angle.

7) Calculate the expected position of a target point; then return to step 2;

8) The end.

Flow chart is shown in Figure 5.

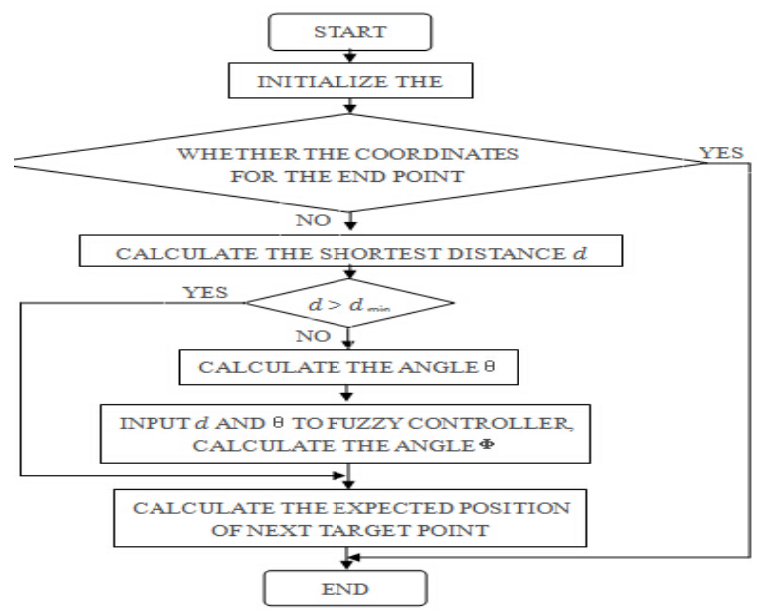

Figure 5. The block diagram of Program

IV. THE RESULTS OF ANALYSIS PLATFORM OF ROBOTIC FISH

Based on MRDS CCR and DSS, URWPGSim2D is established by using $\mathrm{C} / \mathrm{S}$ model, which imitates and 
simulates the entity robotic fish and water environment including environmental information, drive form, movement mode, data stream transmission form, shape, and swimming effect [10].Underwater Robot Water Polo Game Simulator 2D Edition mainly regards the robotic fish's water polo as a formal event: the ball fight, synchronized swimming, and collaboration via hole. These projects complete the mission with the aim of basic obstacle avoidance and path planning.

In this paper, the idea of fuzzy control is applied to the collaboration through holes in the project. In the $2 \mathrm{D}$ platform, fish can reach the designated position, avoid obstacles safely, and plan an optimal path intelligently in a complex environment. As shown in Figure 6 (1), we give the initial position to the two fish in the simulation. The robotic fish in both sides of platform, the robotic fish step is 0.2 , the minimum collision distance is 1 . The goal of the experiments is to realize two fish swapping position, without collision. At the beginning, the robotic fish is at the preliminary position, when the simulation started, the two robotic fish begin to run. When the robotic fish arrive at the first corner, The robotic fish detects obstacles (the wall), it begins to turn the right (left).As shown in Figure 6 (2), Robotic fish perfectly passing the first corner can be seen in this figure. Then robotic fish swim in straight lines, as shown in Figure 6(3), without crashing against the wall. When two robotic fish meet each other, according to the established principles, the robotic fish began to avoid dynamic obstacles. Finally No. 2 fish in the above and No. 1 fish in the below, in this way the fish swim through the intersection, without crashing against the wall, as shown in Figure 6(4). In the end, two robotic fishes safely through the last crossing as shown Figure 6 (6). At last, the two robotic fishes swap position accurately and reach the target point.
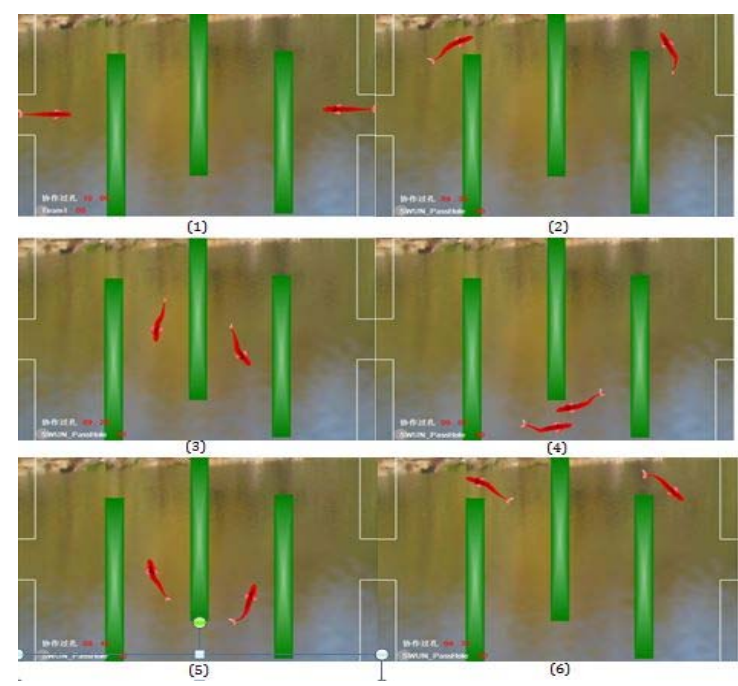

Figure 6.The motion trajectory of robotic fish
Through the actual effect in obstacle avoidance of the robotic fish, it truly gives an index to the rapidity and the superiority of the fuzzy algorithm, and provides the theoretical foundation for our further research in the future.

\section{V.CONCLUSION}

In the path planning of obstacle avoidance, the paper puts forward the algorithm of fuzzy control to plan an optimal path intelligently in direct to complexity and uncertainty of underwater condition. Under the circumstance of ensuring the robotic fish's speed and security, it provides the robotic fish the motion path of real-time and stability, and also has a remarkable effect on all kinds of obstacles. When the obstacle is irregular, it extracts the edge approximately to convert rectangular and concludes the calculation method of the shortest distance between the robotic fish and obstacles, and the angle between them and the target. The simulation experiment proves that not only the fuzzy control theory is feasible with ideal consequence and less amount of calculation, but also it can improve the robotic fish's control accuracy and provide fantastic ideas for the in-depth study of the complicated robotic fish's control strategy.

\section{ACKNOWLEDGEMENTS}

This work was supported by the Science Research Project of Southwest University for Nationalities (Grant No. 2012NFW002)

\section{REFERENCES}

[1] Zhang Jian-guo, Ni Yude, Sharma A B. Data buses takeflight[J]. Circuits and Devices Magazine, IEEE.July 2002:18-31.

[2] ZhengJianlin rocket system bus [D]. transmission technology research of Engineering National University of Defense Technology master thesis control, 2005: 1-4.

[3] Hall I K, Stigall P D. Distributed flight control system using fiber distributed data interface $[\mathrm{J}]$. Aerospace and Electronic Systems Magazine, IEEE. June 1992: 21-33.

[4] Tong Yan, XuDemin, Shi Kyoho. An application to mobile robot path tracking control method of [J]. fire and command control, 2008, 33 (12): $98-101$

[5] Li Qingchun, GaoJunwei, XieGuangming, bionic robotic fish fuzzy control algorithm of obstacle avoidance based on $[\mathrm{J}]$. automation, 2011,30 (12): 65-69.

[6] Fu Yili, GuXiaoyu, Wang Shuguo. Research on the strategy of [J]. robot, an autonomous robot path planning based on fuzzy control, 2004, 26 (6): 548-552.

[7] Wen Sufang, Zhu Qidan, Zhang Xiaofang. Fuzzy controller for mobile robot path planning simulation application based on [J]. technology, 2005 (4): 31-33.

[8] Chen Weidong, Zhu Qiguang. Fuzzy control algorithm for mobile robot path planning algorithm based on [J]. Acta electronica Sinica, 2011 (4): 971-974.

[9] XieChaoping, Kong Feng, Jin Tao. Steering technology and Application Research of [J]. robot control of bionic robotic fish based on fuzzy control, 2009 (4): 26-27.

[10] Ren Jing, XieGuangming. The robotic fish simulation platform of 2D collision detection algorithm [J]. automation, 2011-12 (30): 87-90. 